\title{
Embolismo paradojal - tromboembolismo pulmonar e isquemia cerebral por foramen oval permeable: reporte de caso
}

\author{
Paradoxical embolism - pulmonary thromboembolism and cerebral \\ ischemia due to patent oval foramen, case report
}

\author{
Rafael Tapia Pérez ${ }^{1}$, Julio César Álvarez Gamero² \\ ${ }^{1}$ Médico Asistente, Servicio de Unidad de Cuidados Intensivos, Hospital Nacional Carlos Alberto Seguín Escobedo, EsSalud, Arequipa, \\ Perú. \\ ${ }^{2}$ Médico Cirujano, Centro de Investigación y Estudios Médicos (CIEM).
}

\begin{abstract}
Resumen
El 30 a 40\% de las enfermedades cerebrovasculares presentan un origen desconocido o criptogénico. Puede estar relacionado con la persistencia de foramen oval (FOP), cuya prevalencia es de 12 a 30\%. Reportamos el caso de una paciente mujer de 62 años, que fue intervenida por tumoración de tiroides. En el postoperatorio presentó disnea, hipoxemia, compromiso de conciencia y afasia de expresión. La ecocardiografía transtorácica mostró dilatación de cámaras derechas y la ecocardiografía transesofágica reveló un foramen oval permeable (FOP) con la presencia de ecos compatibles con coágulos que bordeaban dicho orificio: la tomografia cerebral evidenció un infarto cerebral en el hemisferio izquierdo. El hallazgo de coágulos y del FOP en el intraoperatorio confirmó el desarrollo de tromboembolismo pulmonar y embolismo paradojal.

Palabras clave. Embolismo Paradojal; Isquemia Cerebral; Foramen Oval.
\end{abstract}

\section{Abstract}

30 to $40 \%$ of cerebrovascular diseases are of unknown or cryptogenic origin; they may be related to patent foramen ovale (PFO) with a prevalence of 12 to $30 \%$. We report the case of a 62 year old woman who underwent surgery for a thyroid tumor. In the postoperative period she presented dyspnea, hypoxemia, impaired consciousness and expression aphasia. Transthoracic echocardiography showed dilatation of right chambers, and transesophageal echocardiography revealed a patent foramen ovale (PFO) with the presence of echoes compatible with clots bordering this defect; the brain scan revealed a stroke in the left hemisphere. The presence of clots and PFO intraoperatively confirmed the development of pulmonary embolism and paradoxical embolism.

Keywords. Paradoxical Embolism, Cerebral Ischemia, Foramen Ovale.

\section{An Fac med. 2016;77(4):417-20 / http://dx.doi.org/10.15381/anales.v77i4.12661}

\section{INTRODUCCIÓN}

La enfermedad cerebrovascular (ECV) es la tercera causa de muerte y la primera causa de invalidez en el mundo. Aproximadamente 25\% de los varones y $20 \%$ de las mujeres sufrirán una ECV si viven hasta los 85 años o más ${ }^{(1)}$. También presenta alta probabilidad de recurrencia, por lo cual el diagnóstico etiológico es la base para la prevención secundaria. Pero, algunos estudios llegan a reportar un $30 \%$ a $40 \%$ de ECV de causa desconocida o criptogénica, o también llamados stroke criptogénicos (SC) que se presentan principalmente en menores de 55 años, en los que el FO permeable es un hallazgo relativamente común y que se relacionan con embolismo paradojal (EP) ${ }^{(2-4)}$. El embolismo conlleva a stroke o ataque isquémico (derrame cerebral) que puede originarse en el sistema venoso (embolismo paradojal) o en la circulación arterial sistémica ${ }^{(2-5)}$.
El foramen oval persistente (FOP) y el aneurisma septal auricular están asociados a derrame cerebral criptogénico, sugiriendo asociación al embolismo paradojal; sin embargo, no existe relación lineal de causa-efecto. De hecho, la presencia de un defecto como el FOP no constituye una relación lineal como factor de riesgo independiente de derrame cerebral isquémico ${ }^{(1,2,5,6)}$. El derrame cerebral cripotogénico es determinado clínicamente y por neuroimagen; se debe plantear estudios de 
ecocardiografía transtorácica (ETT) o ecocardiografía transesofágica (ETE), y buscar fuentes de formación de coágulos o de defectos en la coagulación.

Reportamos el caso de una paciente que presentó un stroke isquémico por embolismo paradojal con foramen oval permeable secundario a una trombosis venosa profunda complicado con embolia pulmonar.

\section{CASO CLÍNICO}

Paciente mujer, de 62 años, que ingresó al Hospital Nacional Carlos Alberto Seguín Escobedo, EsSalud, Arequipa, Perú, para tratamiento quirúrgico de tumoración de tiroides derecha. Tenía como antecedente 18 años atrás de una hemitiroidectomía izquierda por tumoración de glándula tiroidea. En este ingreso se le realizó hemitiroidectomía derecha. A los dos días de la operación, presentó dolor en extremidades inferiores, náuseas y vómitos. Un día después tuvo desorientación, excitación psicomotriz y dificultad respiratoria. Progresivamente se observó hipoxemia, tendencia a hiposaturación de oxígeno (SaO2 83\%). Posteriormente mostró compromiso del estado de conciencia, llegando al sopor, afasia de expresión, sin compromiso motor de extremidades derechas. Se le realizó tomografía cerebral, no apreciándose lesión estructural inicial.

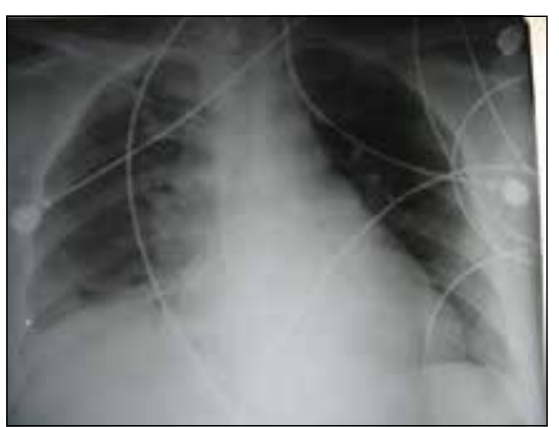

Figura 1. Radiografía de tórax, en la que se aprecia empastamiento parahiliar y velamiento basal derecho.

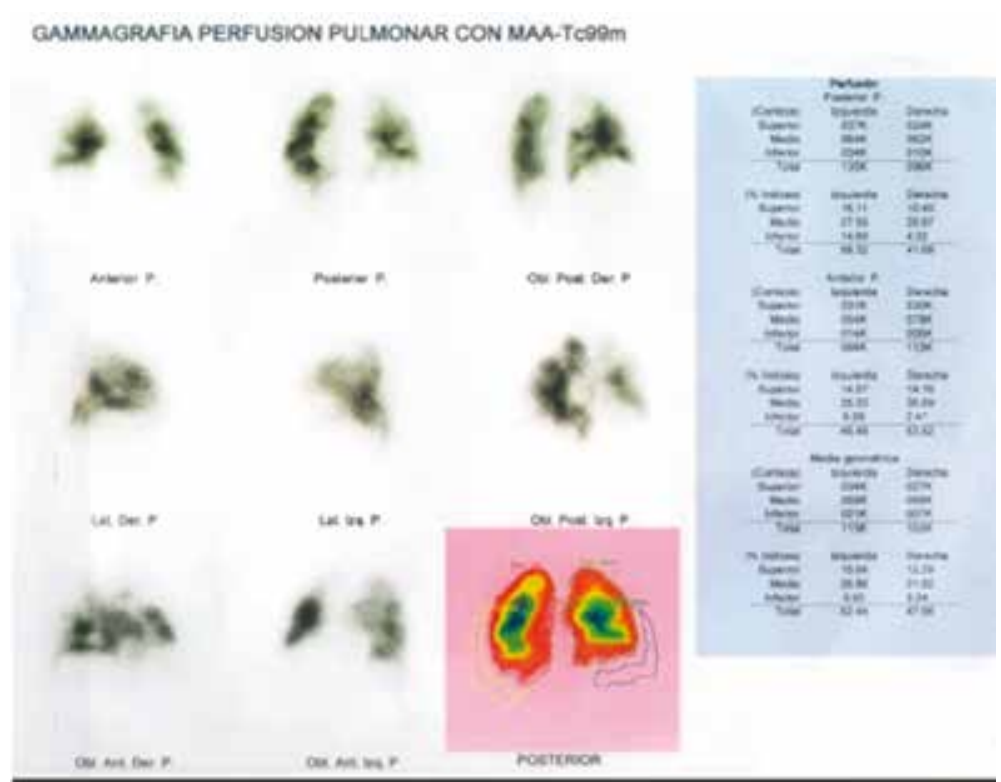

Figura 2. Gammagrafía de la perfusión pulmonar.

Fue transferida a cuidados intensivos y se planteó la posibilidad de un cuadro neurológico y respiratorio. La ecocardiografía transtorácica (ETT) mostró dilatación de las cavidades derechas, sobretodo de ventrículo derecho. La ecografía Doppler de carótidas fue sin particularidades y la de miembros inferiores reveló signos compatibles con trombosis venosa profunda (TVP). La radiografía de tórax mostró elevación del hemidiafragma derecho (figura 1). La gammagrafía de perfusión pulmonar (figura 2) evidenció un patrón extenso de falta de perfusión en ambos pulmones. Estos estudios plantearon el diagnóstico de tromboembolismo pulmonar, por lo que se inició tratamiento con heparina. Sin embargo, la paciente continuó con afasia y hemiparesia facial derecha. Se repitió la tomografía cerebral a las 24 horas, y se observó una imagen hipodensa extensa que comprometía región fronto-parietal izquierda, compatible con infarto cerebral (figura 3).

Por los hallazgos neurológicos y cardiorrespiratorios y la probabilidad de una relación temporal y causal en estos eventos, se realizó ecocardiografía transesofágica (ETE) (figura 4), que reveló una comunicación interauricular com- patible con (FOP) bordeado de imagen compatible con coágulo. La paciente fue intervenida quirúrgicamente y se le realizó el cierre de defecto del septum auricular y embolectomía. Posteriormente, presentó un postoperatorio compensado, quedando con afasia de expresión, hemiparesia facial izquierda. Recibió tratamiento anticoagulante con heparina de peso molecular bajo, pasando a piso 5 días después.

\section{DISCUSIÓN}

El foramen oval permeable es identificado mediante una ETE en aproximadamente $25 \%$ de personas saludables,

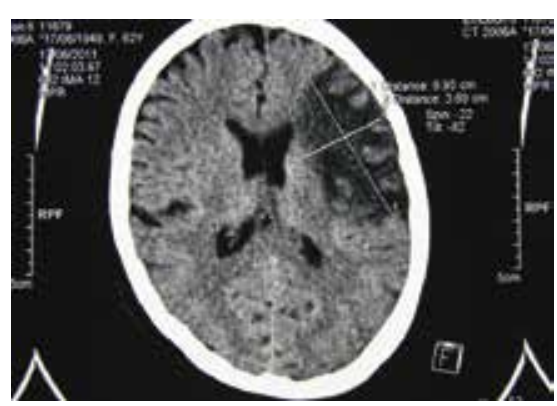

Figura 3. Tomografía cerebral compatible con stroke isquémico. 


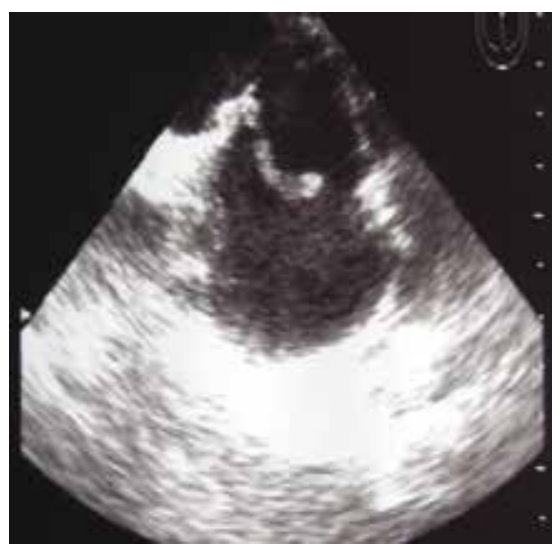

Figura 4. Ecografía transesofágica que confirma la presencia de foramen oval permeable y se aprecia una imagen compatible con coágulo.

y en autopsias constituye un hallazgo hasta de 35\% ${ }^{(1,5,14)}$. EL FOP es generalmente benigno, pero ha sido asociado a derrame cerebral, migraña, síndrome platipnea-ortodesoxia y a enfermedad embólica por descompresión del buceador. Aproximadamente 30\% a 40\% de las enfermedades cerebrovasculares isquémicas (ECV) son criptogénicas, es decir, sin causa aparente. La asociación entre FOP e infarto cerebral criptogénico o stroke criptogénico (SC) sigue siendo controvertida, pues los estudios son aún contradictorios ${ }^{(7)}$. Los estudios que indican esta asociación postulan diferentes mecanismos implicados:

a. Embolia paradójica, con el paso de trombos desde el sistema venoso periférico a cavidades cardiacas izquierdas a través del FOP;

b. Formación de trombos en las aurículas como consecuencia de arritmias relacionadas con el FOP;

c. Formación de trombos en el canal del foramen oval; $y$,

d. Estados de hipercoagulabilidad relacionados con el FOP (7). En el estudio PICCS, Homma y col. encontraron que los defectos y cortocircuitos más grandes presentaban un mayor riesgo de infarto criptogénico ${ }^{(8,9)}$. Otros factores de riesgo parecen ser el cortocircuito espontáneo en reposo, sin maniobras de valsalva, una separación entre el septum primum y el septum secundum mayor de $5 \mathrm{~mm}$ o la presencia de aneurisma del septo interauricular (ASI) ${ }^{(7)}$.

En la mayoría de los casos, la presencia de FOP es un hallazgo casual sin repercusiones clínicas. El tratamiento de elección del FOP todavía no está definido. Así, se recomienda no dar tratamiento alguno en los casos asintomáticos y agregar antiplaquetarios o anticoagulantes en caso de trombos en cavidades cardiacas, fenómenos trombofílicos asociados o episodios previos de derrame cerebral ${ }^{(5,10-12)}$.

Según estudios observacionales, la probabilidad de que el FOP participe en la ECV aparece asociada a dos condiciones: la edad y las características anatómicas de la malformación (3). Así como también, la predisposición a trombogénesis.

La asociación entre FOP y ECV es diferente según la edad de los pacientes. Mientras en los que presentan ECV antes de los 55 años la incidencia de FOP supera el $50 \%$ comparado a un 10 $20 \%$ en los controles, en los de mayor edad no se demuestra una diferencia significativa ${ }^{(3)}$. Así en un metaanálisis de casos-control, Overell y col. no encontraron asociación entre FOP y ECV en los mayores de 55 años (OR 2,26; $95 \%$ IC: 0,96 a 5,31 ), pero era significativa en los menores de 55 años, con un OR de 6,0 (95\% IC 3,72 a 9,68) ${ }^{(13)}$.

El caso que reportamos tiene peculiaridades interesantes, porque se trata en primer lugar de una paciente con neoplasia de tiroides, intervenida quirúrgicamente; no recibió profilaxis antitrombótica, y a los dos días presentó dolor en miembros inferiores, síntomas que no fueron interpretados correctamente. Al siguiente día mostró repentinamente dificultad respiratoria, hipoxemia y compromiso progresivo del estado de conciencia, detectando afasia de expresión con hemiparesia facial izquierda. Es decir, que previo a los síntomas neurológicos, se puede establecer un problema respiratorio agudo que debió hacer sospechar en tromboembolismo pulmonar (TEP). Esto se confirmó con la ETT, la gammagrafía de perfusión pulmonar (figura 2) y el ecodoppler de miembros inferiores. Sin embargo, no explica el compromiso de conciencia y los hallazgos tomográficos del cerebro que fueron compatibles con infarto cerebral o stroke isquémico (figura 3). Es la ETE la que confirmó la presencia de FOP y en donde puede observarse además una imagen flotante compatible con un coágulo (figura 4).

En este caso puede deducirse una serie de hechos que conllevaron a la paciente a un fenómeno tromboembólico paradojal que nació con la formación de trombos en el sistema venoso profundo. El concepto de criptogénico es cuestionable en este caso, por la bibliografía revisada, porque la tecnología nos acercó rápidamente a una etiología con una secuencia fisiopatológica deliberadamente lógica.

La paciente, luego de la corrección quirúrgica del FOP, de la embolectomía cardiaca y del manejo con heparina, fue estabilizada quedando como secuela afasia de expresión y hemiparesia facial izquierda.

\section{REFERENCIAS BIBLIOGRÁFICAS}

1. Taboada Caballero E, Peñaranda Villagomez M Embolismo paradojal (caso clínico). Rev Inst Méd Su. 2009;8(1):27-30.

2. Fontanella B, Vizzardi E, Bordonali T, D'Aloia A, Chiari E, et al. Pulmonary embolism complicated by impending paradoxical embolism - a case report and review of the literature. Kardiol Pol. 2010;68(3):314-6.

3. Martinez A, Heusser F. Accidente vascular encefálico y foramen oval permeable. Boletín Escuela de Medicina UC, Pontificia Universidad Católica de Chile. 2006;31(1):24-8.

4. Mesa D, Franco M, de Lezo JS, Muñoz J, Rus C, Delgado $\mathrm{M}$, et al. Prevalencia de foramen oval permeable en pacientes jóvenes con accidente isquémico cerebral de causa desconocida. Rev Esp Cardiol. 2003;56(7):662-8.

5. Prabhakaran S, Elkind M. Cryptogenic stroke. UpToDate, Waltham, MA. Disponible en: http:// www.uptodate.com/contents/cryptogenic-stroke. Acceso el 17 de abril 2016.

6. Veltri MA, Pérez MH, Soloaga ED, Chertcoff FJ, Manuale O, Ubaldini JE. Embolia paradojal inminente. Medicina. 2006;66(6):558-560.

7. Cruz-González I, Solis J, Inglessis-Azuaje I, Palacios IF. Foramen oval permeable: situación actual, Rev Esp Cardiol. 2008;61(7):738-51.

8. Homma S, Sacco RL. Patent foramen ovale and stroke. Circulation. 2005;112:1063-72. 
9. Homma S, Sacco RL, Di Tullio MR, Sciacca RR, Mohr JP. Effect of medical treatment in stroke patients with patent foramen ovale: patent foramen ovale in the Cryptogenic Stroke Study. Circulation. 2002;105:2625-31.

10. Thaler D, Kent D. Rethinking trial strategies for stroke and patent foramen ovale. Curr Opin Neurol. 2010 February;23(1):73-8.

11. Lange MC, Zamproni LN, Braatz V, Fernandes AF, Zetola VF, Nóvak EM. Ischemic stroke in a patient with Crohn's disease: a confirmed paradoxical embolism mechanism, Arq Neur-Psiquiatr. Aug 2010;68(4):651-2.

12. Mousavi N, Jassal DS, Moon MC, Shaikh N, Soni A. Thrombus in transit. Can J Cardiol. 2010 March;26(3):133.
13. Overell JR, Bone I, Lees KR. Interatrial septal abnormalities and stroke-a meta-analysis of casecontrol studies. Neurology. 2000;55:1172-9.

14. Torbey E, Thompson PD. Patent foramen ovale: thromboembolic structure or incidental finding? Conn Med. 2011 Feb;75(2):97-105.

15. Erkut B, Sevimli S, Ates A, Erdem AF, Dogan N, Kantarci M. Entrapped thrombus in a patent foramen ivale complicated by pulmonary embolism without paradoxical embolism. Tex Heart Indt J. 2008;35(3):371.

Artículo recibido el 11 de mayo de 2016 y aceptado para publicación el 7 de julio de 2016.
Declaración:

Este trabajo de investigación no ha sido publicado previamente o remitido a otra revista biomédica.

Financiación y conflicto de intereses.

No hay ningún tipo de financiación. No hay conflicto de intereses.

Autor corresponsal:

Dr. Rafael Fredy Tapia Pérez.

Dirección del autor: Urb. Las Condes B-8, Sachaca,

Arequipa.

Teléfono/Fax: 959654627 - 253937

Correo electrónico: rafaeltapiap@gmail.com 